\title{
RT-PCR detection of porcine reproductive and respiratory syndrome virus based on the ORF5 gene in mainland China, 2012-2015
}

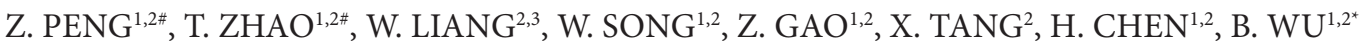

\begin{abstract}
${ }^{1}$ State Key Laboratory of Agricultural Microbiology; College of Animal Science and Veterinary Medicine, Huazhong Agricultural University, Wuhan, 430070, P. R. China; ${ }^{2}$ The Cooperative Innovation Center for Sustainable Pig Production, Huazhong Agricultural University, Wuhan, 430070, P. R. China; ${ }^{3}$ Key Laboratory of Animal Genetics, Breeding and Reproduction, Ministry of Education; College of Animal Science and Veterinary Medicine, Huazhong Agricultural University, Wuhan, 430070, P. R. China
\end{abstract}

\begin{abstract}
Summary. - Between January 2012 and December 2015, 13,567 clinical samples were collected from healthy pigs and pigs with a history of respiratory symptoms and/or reproductive disorders in 29 provinces of mainland China to detect porcine reproductive and respiratory syndrome virus (PRRSV). From these samples, 7490 were PRRSV-positive (average value, 55.21\%). The annual PRRSV detection rate from 2012 to 2015 for each year is $45.67 \%, 55.99 \%, 56.91 \%$, and 59.07\%, respectively. Phylogenetic analyses revealed that the percentage of highly pathogenic PRRSV strains had a decreasing yearly trend, whereas the vaccine-like strains showed the opposite trend during the years 2012-2015. These data indicate that while the vaccine contributes to PRRSV prevention in China, it might also lead to serious problems. Specifically, we identified 12 NADC30-like PRRSVs during the years 2014-2015, suggesting potential transmission of the newly emerged PRRSV strains in China. Our data contribute to new information about the epidemiology of PRRSV.
\end{abstract}

Keywords: porcine reproductive and respiratory syndrome virus; recent profile; RT-PCR detection; ORF5 gene; China

Porcine reproductive and respiratory syndrome (PRRS) has been prevalent worldwide for almost 30 years since it was first reported in the USA in 1987. It has become one of the most economically significant diseases in the global pig farming industry (Han and Yoo, 2014). The causative agent of PRRS, the porcine reproductive and respiratory syndrome virus (PRRSV), is a single-stranded, positive-sense, enveloped RNA virus that belongs to the genus Arterivirus, the family Arteriviridae (Cavanagh, 1997). Two PRRSV genotypes have been defined based on the genomic heterogeneity as type 1 , which comprises of the European type isolates represented by the Lelystad virus (LV), and type 2, which includes the North

*Corresponding author. E-mail: wub@mail.hzau.edu.cn or hzauwubin@163.com; phone: +86-27-87287201."These two authors contributed equally to this work.

Abbreviations: $\mathrm{LV}=$ Lelystad virus; NA type $=$ North America type; PRRS = porcine reproductive and respiratory syndrome; PRRSV $=$ PRRS virus
American (NA) type isolates represented by VR-2332 (Martínez-Lobo et al., 2011). These genotypes share only 55-70\% nucleotide sequence identity (Forsberg et al., 2002; Han and Yoo, 2014). The PRRSV genome possesses ten open reading frames (ORFs). Of these, ORF1a and ORF1b encode ppla and pplab, which are two non-structural polyproteins from which at least 16 non-structural proteins including nsp $1 \alpha$, nsp1 $\beta$, nsp2, nsp2N, nsp2TF, nsp3-6, nsp7 $\alpha$, nsp7 $\beta$, nsp8-12 are derived, while ORF2 to ORF7 encode the following eight structural proteins: GP2a, E (2b), GP3, GP4, 5a, GP5, M, and $\mathrm{N}$ (Chen et al., 2016). Among these ORFs, the ORF5 gene, which encodes the major envelope protein GP5, is one of the most variable regions in the PRRSV genome (Allende et al., 2000; Zhou et al., 2015), and is proposed therefore to be an optimal region for monitoring the evolution of PRRSV (Shi et al., 2010; Li et al., 2011; Xie et al., 2014).

Nine years after PRRS emerged in the USA, PRRSV was first reported in China in 1996, and the infections resulting from this Arterivirus have now become one of the gravest 
threats to the country's pig herds, causing enormous economic losses each year (Xie et al., 2014). The 2006 atypical PRRS outbreak in China led to over 2,000,000 cases of infection, around 400,000 fatal cases, and more than 1,2 billion in Chinese Yuan in direct economic losses (Tian et al., 2007). Several variants of PRRSV strains such as JXA1, HEB1, HUB2 and HuN4 were proposed to be responsible for this outbreak (Tian et al., 2007; Tong et al., 2007). Similarly, the more recent widespread outbreaks of PPRS in several parts of China in 2014 are likely to be associated with the novel PRRSV NADC30-like strain, CHsx1401 (Zhou et al., 2015). Therefore, there is a pressing need to monitor the epidemic dynamics of this virus.

Between the years 2012 and 2015, a total of 13,567 clinical samples (lungs, kidneys, lymph nodes, tonsils and serum) comprising 2,492 samples in 2012, 3,424 samples in 2013, 3,915 samples in 2014, and 3736 samples in 2015 from pigs with a history of respiratory symptoms and/or reproductive disorders were collected from 29 provinces in mainland China, excluding Tibet and Ningxia (Fig. 1). The solid samples were homogenized using QIAGEN TissueLyser II (Qiagen, Germany) followed by total RNA extraction using an OMEGA Total RNA kit I (Omega, USA). Viral cDNA, used as the template in the RT-PCR (Li et al., 2015), was synthesized using MMLV reverse transcriptase (Takara, Japan). A pair of primers targeting the ORF5 gene (forward sequence from 5' to 3': GAG GTGGGCAACYGTTTTAG; reverse sequence from 5 ' to 3': CAMGMGTAGCGCCAGGACA) was designed and synthesized (BGI, China). Thermocycler conditions used for PCR were $5 \mathrm{~min}$ at $95^{\circ} \mathrm{C}$, followed by 35 cycles of 30 $\mathrm{s}$ at $95^{\circ} \mathrm{C}, 30 \mathrm{~s}$ at $58^{\circ} \mathrm{C}$ and $1 \mathrm{~min}$ at $72^{\circ} \mathrm{C}$, and a final extension of $10 \mathrm{~min}$ at $72^{\circ} \mathrm{C}$. The products were analysed on $1 \%$ gel electrophoresis. After that, the entire ORF5 sequences of all the PRRSV-positive samples were amplified using another pair of primers (F: GGCGACCGTTTTAGCCT GTCTT; R: ATCATTATTGGCGTGTAGGTG) using the same thermocycler conditions mentioned above and sequenced for further analysis. The products were firstly separated by electrophoresis on a $1 \%$ agarose gel, and then purified using a TIANgel midi purification kit (Tiangen, China) following the manual instructions and then cloned into a PMD18-T vector (Takara). Plasmids recovered from the positive colonies were further confirmed by DNA se-

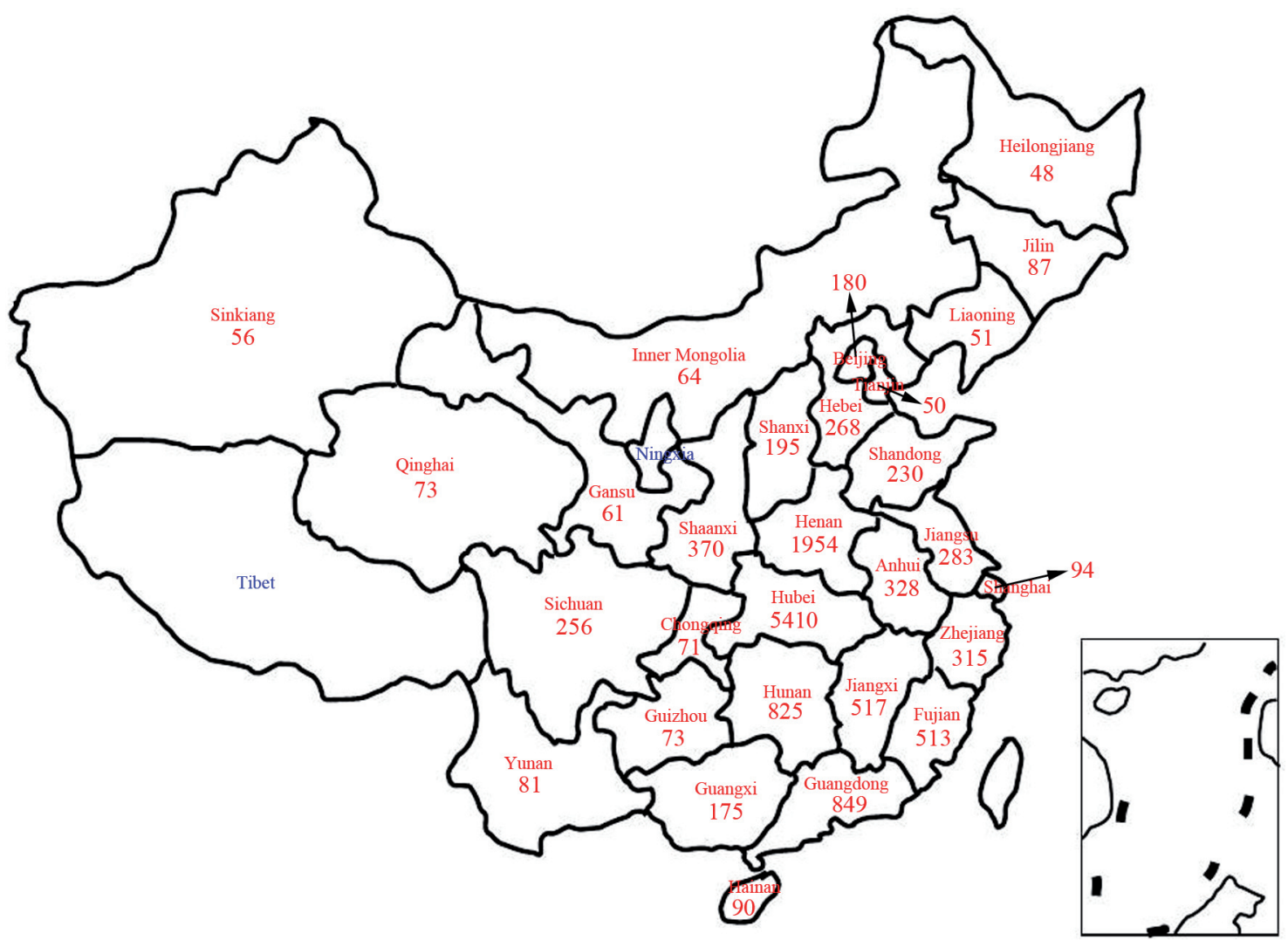

Fig. 1

Distribution of clinical samples collected from the 29 provinces in mainland China 


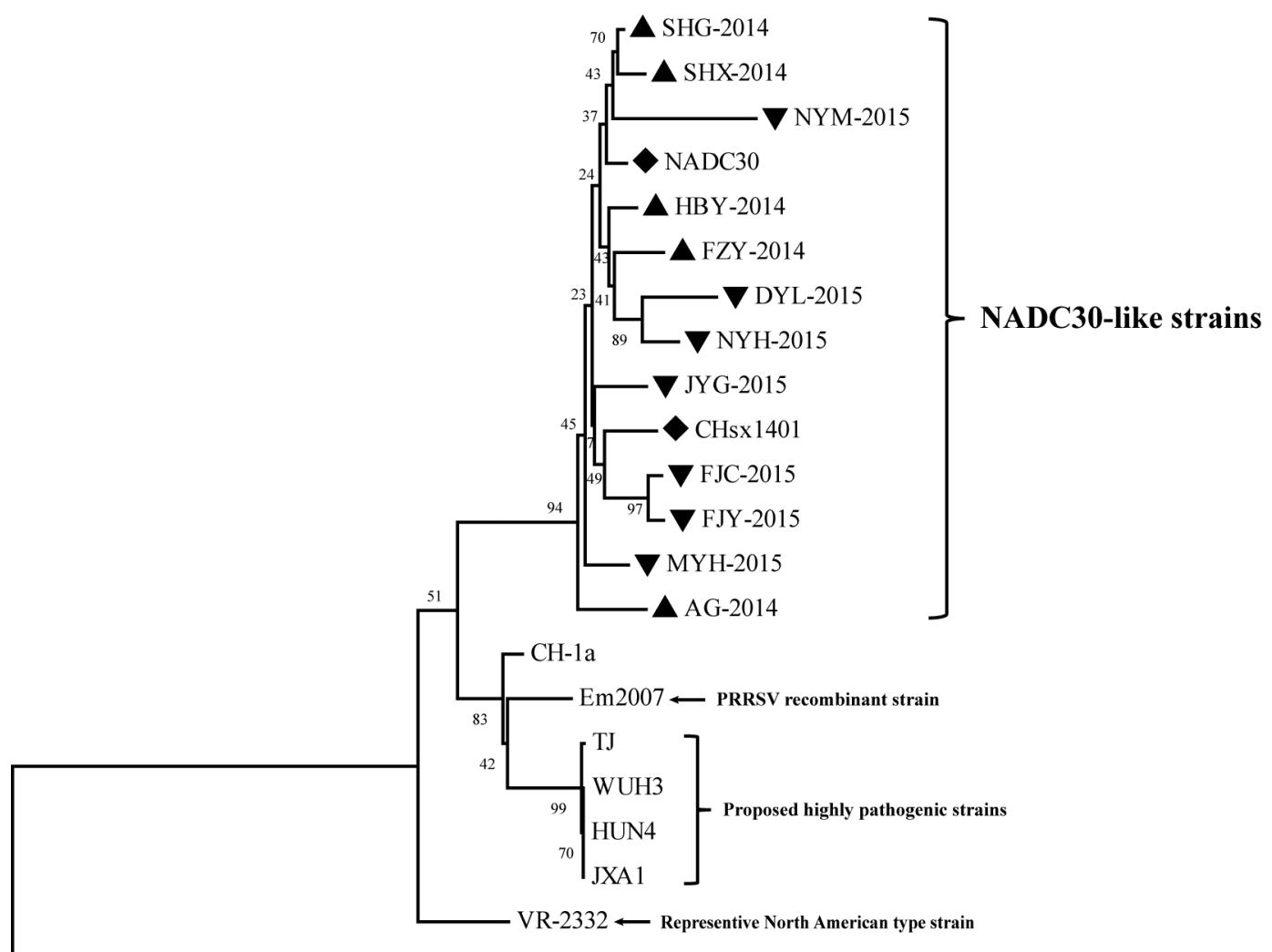

Lelystad virus $\longleftarrow$ Representive European type strain

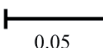

Fig. 2

Phylogenetic analyses of the ORF5 genes of the 12 PRRSV NADC30-like strains derived from this study

" $\mathbf{\Delta}$ " represents the NADC30-like strains determined in 2014 and " $\boldsymbol{\nabla}$ " indicates the NADC30-like strains identified in 2015. Representative prototype strains CHsx1401 (GenBank Acc. No. KP861625), TJ (EU860248), WHU3 (HM853673), HUN4 (EF635006), JAX1 (EF112445), Em2007 (EU262603), and CH1a (AY032626) were isolated from China. Of which PRRSV strain CHsx1401 is the novel NADC30-like strain reported in China in 2015 (Zhou et al., 2015); while Em2007 is reported as a PRRSV recombinant strain between PRRSV vaccine strains and PRRSV circulating strains (Li et al., 2009a). Representative prototype strains NADC30 (JN654459) and VR2332 (AY150564) were isolated from the United States. Prototype Lelystad virus (M96262) was used as the out group. The phylogenetic tree was constructed by using the distance-based neighbor-joining method with 1,000 bootstrap replicates in MEGA6. Numbers along branches are bootstrap values. Scale bar indicates nucleotide substitutions per site.

quencing (BGI). The assembled sequence data, as well as the publicly available ORF5 sequences of PRRSV strains from GenBank (http://www.ncbi.nlm.nih.gov/genbank/) were used for the phylogenetic analysis. Multiple sequence alignments were performed using ClustalW (Thompson et al., 2002). A phylogenetic tree was generated by MEGA 6.0 software (Tamura et al., 2013) using the neighbor-joining algorithm with 1,000 bootstraps based on the comparative results exported from ClustalW.

From these samples, approximately 7,490 of the total samples investigated here were positive for PRRSV, as based on detection of the ORF5 gene, with an average positive rate of 55.21\% $(7,490 / 13,567)$. Annually, the PRRSV detection rate over the study period was $45.67 \%$ (1138/2492, 2012),
$55.99 \%(1917 / 3424,2013), 56.91 \%(2228 / 3915,2014)$, and $59.07 \%(2207 / 3736,2015)$, respectively, with an increasing yearly trend. Monthly, the highest positivity rates occurred in January, February, March and November. Seasonally, winter (December, January and February) is the season with a highest PRRSV detection rate over the past four years, while the highest values for the other seasons, in descending order, were autumn (September, October and November), summer (June, July and August) and spring (March, April and May) (Supplementary Table S1). For geographical distribution, the PRRSV detection rates in Northeast China, East China and South China were higher than for the other parts of China during the past four years (Supplementary Table S2). 
The entire ORF5 sequences of the 7,490 PRRSV-positive samples were amplified and sequenced for phylogenetic analysis, which showed that all of the 7,490 strains were closely related to the NA type representative VR-2332 but far from the European type representative LV, suggesting that the current epidemic PRRSV in China is mainly the NA type virus. This finding is consistent with Li et al. (2011) who performed an epidemiological investigation of PRRSV in China between 2006 and 2010. Among the 7,490 strains, $27.36 \%$ of them was found to have close relationships with the ORF5 genes of the highly pathogenic PRRSV strains described previously (e.g., PRRSV strains HuN4 (Tong et al., 2007), TJ (Leng et al., 2008), JXA1 (Tian et al., 2007), HEB1 (Tian et al., 2007) and WuH3 (Li et al., 2009b)), while 56.08\% were close to the PRRSV vaccine strains (e.g., TJM (Leng et al., 2012), JXA1-R (Leng et al., 2012), HuN4-F112 (Tian et al., 2009)). However, only $3.60 \%$ of the PRRSVs were similar to the classical NA PRRSV-type strains. The results suggest that the PRRSVs circulating in China in recent years are mainly the highly pathogenic-like strains. However, the highly pathogenic-like PRRSV positivity rate displayed a yearly decreasing trend $(64.52 \%$ in $2012,22.17 \%$ in $2013,17.78 \%$ in 2014 , and $20.00 \%$ in 2015). In comparison, detection of the vaccine-like strain increased annually $(24.73 \%$ in 2012 , $65.65 \%$ in $2013,64.07 \%$ in 2014 , and $59.53 \%$ in 2015 ). These findings indicate that wide use of PRRSV vaccines in China has helped to prevent PRRS, but the large scale use of these vaccines is becoming a serious problem, as was also revealed by the year-to-year percentage increase in the numbers of PRRSV recombinants between the vaccine strains and the circulating viruses. Indeed, the annual recombinant PRRSV strain prevalence from 2012 to 2015 was $16.74 \%, 20.70 \%$, $24.30 \%$, and $38.35 \%$, respectively. In particular, 12 PRRSVs (5 obtained from 2014 and 7 obtained from 2015) are located in the same branch as the novel PRRSV NADC30 strain (Fig. 2). The PRRSV NADC30 strain, which was isolated in the USA in 2008, is defined as a highly pathogenic strain that can quickly induce viremia in infection-challenged pigs compared with other PRRSV isolates (Brockmeier et al., 2012). The NADC30-like PRRSV in China, which was first reported in 2015, was proposed to be associated with severe outbreaks of PRRS in some intensive pig farms in China from August to December 2014 (Zhou et al., 2015). The finding that 12 PRRSVs are genetically close to NADC30 PRRSV suggests the future potential for higher prevalence in the newly emerged NADC30-like PRRSV strains found in Chinese pig herds.

To conclude, the present study briefly reports the situation for PRRSV detection in China in the past four years (20122015). From these data we deduce that even the PRRSV strains circulating in Chinese pig herds in recent years are mainly the highly pathogenic-like strains, yet the prevalence of PRRSV has been restrained effectively, and this might be largely related to the wide use of PRRSV vaccines. However, relying on vaccines to prevent the spread of PRRSV and outbreaks of PRRS may also lead to serious problems. Attention should be paid especially to the potential prevalence of the newly emerged NADC30-like PRRSV strains in Chinese pigs, which may increase the difficulty of controlling PRRSV. The data retrieved from the present study will contribute to better understanding of the current prevalence of PRRSV in China.

Acknowledgements. This study was supported by the National Key Technology Research and Development Program of the Ministry of Science and Technology of China (grants 2015BAD12B04 and 2014BAD20B01), and the Foundation for Innovative Research Groups of the National Natural Science Foundation of China (grant number 31421064).

Supplementary information is available in the online version of the paper.

\section{References}

Allende R, Kutish G, Laegreid W, Lu Z, Lewis T, Rock D, Friesen J, Galeota J, Doster AR, Osorio F (2000): Mutations in the genome of porcine reproductive and respiratory syndrome virus responsible for the attenuation phenotype. Arch. Virol. 145, 1149-1161. https://doi.org/10.1007/ $\underline{\text { s007050070115 }}$

Brockmeier SL, Loving CL, Vorwald AC, Kehrli ME, Baker RB, Nicholson TL, Lager KM, Miller LC, Faaberg KS (2012): Genomic sequence and virulence comparison of four Type 2 porcine reproductive and respiratory syndrome virus strains. Virus Res. 169, 212-221. https://doi. org/10.1016/j.virusres.2012.07.030

Cavanagh D (1997): Nidovirales: a new order comprising Coronaviridae and Arteriviridae. Arch. Virol. 142, 629-633.

Chen N, Trible BR, Kerrigan MA, Tian K, Rowland RR (2016): ORF5 of porcine reproductive and respiratory syndrome virus (PRRSV) is a target of diversifying selection as infection progresses from acute infection to virus rebound. Infect. Genet. Evol. 40, 167-175. https://doi.org/10.1016/j. meegid.2016.03.002

Forsberg R, Storgaard T, Nielsen HS, Oleksiewicz MB, Cordioli P, Sala G, Hein J, Bøtner A (2002): The genetic diversity of European type PRRSV is similar to that of the North American type but is geographically skewed within Europe. Virology 299, 38-47. https://doi.org/10.1006/ viro.2002.1450

Han M, Yoo D (2014): Engineering the PRRS virus genome: Updates and perspectives. Vet. Microbiol. 174, 279-295. https:// doi.org/10.1016/j.vetmic.2014.10.007

Leng X, Li Z, Xia M, Li X, Wang F, Wang W, Zhang X, Wu H (2012): Mutations in the genome of the highly pathogenic porcine reproductive and respiratory syndrome virus potentially related to attenuation. Vet. Microbiol. 157, 50-60. https:// doi.org/10.1016/j.vetmic.2011.12.012 
Leng X, Wen Y, Qi Q, Li Z, Wu H (2008): Isolation and identification of the very virulent porcine reproductive and respiratory syndrome virus (PRRSV) TJ strain. J. Jilin Agricult. Univ. 6, 023.

Li B, Fang L, Guo X, Gao J, Song T, Bi J, He K, Chen H, Xiao S (2011): Epidemiology and evolutionary characteristics of the porcine reproductive and respiratory syndrome virus in China between 2006 and 2010. J. Clin. Microbiol. 49, 3175-3183. https://doi.org/10.1128/JCM.00234-11

Li B, Fang L, Xu Z, Liu S, Gao J, Jiang Y, Chen H, Xiao S (2009a): Recombination in vaccine and circulating strains of porcine reproductive and respiratory syndrome viruses. Emerg. Infect. Dis. 15, 2032-2035. https://doi.org/10.3201/ eid1512.090390

Li B, Xiao S, Wang Y, Xu S, Jiang Y, Chen H, Fang L (2009b): Immunogenicity of the highly pathogenic porcine reproductive and respiratory syndrome virus GP5 protein encoded by a synthetic ORF5 gene. Vaccine 27, 1957-1963. https:// doi.org/10.1016/j.vaccine.2009.01.098

Li B, Wang H, Peng Z, Hu R, Wu B (2015): Genetic variation analysis of Nsp2 and GP5 amino acids of porcine reproductive and respiratory syndrome virus in clinical in central China. China Anim. Husb. Vet. Med. 42, 3026-3036.

Martínez-Lobo FJ, Díez-Fuertes F, Segalés J, García-Artiga C, Simarro I, Castro JM, Prieto C (2011): Comparative pathogenicity of type 1 and type 2 isolates of porcine reproductive and respiratory syndrome virus (PRRSV) in a young pig infection model. Vet. Microbiol. 154, 58-68. https://doi.org/10.1016/j.vetmic.2011.06.025

Shi M, Lam TT, Hon C, Murtaugh MP, Davies PR, Hui RK, Li J, Wong LT, Yip C, Jiang J (2010): Phylogeny-based evolutionary, demographical, and geographical dissection of North American type 2 porcine reproductive and respira- tory syndrome viruses. J. Virol. 84, 8700-8711. https:// doi.org/10.1128/JVI.02551-09

Tamura K, Stecher G, Peterson D, Filipski A, Kumar S (2013): MEGA6: molecular evolutionary genetics analysis version 6.0. Mol. Biol. Evol. 30, 2725-2729. https://doi. org $/ 10.1093 / \mathrm{molbev} / \mathrm{mst} 197$

Thompson JD, Gibson T, Higgins DG (2002): Multiple sequence alignment using ClustalW and ClustalX. Curr. Prot. in Bioinform. 2.3. 1-2.3. 22. https://doi. org/10.1002/0471250953.bi0203s00

Tian K, Yu X, Zhao T, Feng Y, Cao Z, Wang C, Hu Y, Chen X, Hu D, Tian X (2007): Emergence of fatal PRRSV variants: unparalleled outbreaks of atypical PRRS in China and molecular dissection of the unique hallmark. PloS One 2, e526. https://doi.org/10.1371/journal.pone.0000526

Tian Z, An T, Zhou Y, Peng J, Hu S, Wei T, Jiang Y, Xiao Y, Tong G (2009): An attenuated live vaccine based on highly pathogenic porcine reproductive and respiratory syndrome virus (HPPRRSV) protects piglets against HP-PRRS. Vet. Microbiol. 138, 34-40. https://doi.org/10.1016/j.vetmic.2009.03.003

Tong G, Zhou Y, Hao X, Tian Z, An T, Qiu H (2007): Highly pathogenic porcine reproductive and respiratory syndrome, China. Emer. Infect. Dis. 13, 1434-1436. https://doi. org/10.3201/eid1309.070399

Xie J, Cui T, Cui J, Chen Y, Zhang M, Zhou P, Deng S, Su S, Zhang G (2014): Epidemiological and evolutionary characteristics of the PRRSV in Southern China from 2010 to 2013. Microb. Pathog. 75, 7-15. https://doi.org/10.1016/j. micpath.2014.08.001

Zhou L, Wang Z, Ding Y, Ge X, Guo X, Yang H (2015): NADC30like strain of porcine reproductive and respiratory syndrome virus, China. Emerg. Infect. Dis. 21, 2256. https:// doi.org/10.3201/eid2112.150360 


\title{
Supplementary information
}

\section{RT-PCR detection of porcine reproductive and respiratory syndrome virus based on the ORF5 gene in mainland China, 2012-2015}

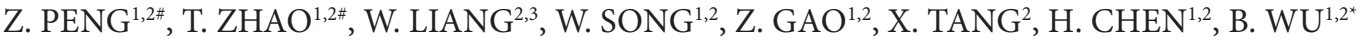

${ }^{1}$ State Key Laboratory of Agricultural Microbiology; College of Animal Science and Veterinary Medicine, Huazhong Agricultural University, Wuhan, 430070, P. R. China; ${ }^{2}$ The Cooperative Innovation Center for Sustainable Pig Production, Huazhong Agricultural University, Wuhan, 430070, P. R. China; ${ }^{3}$ Key Laboratory of Animal Genetics, Breeding and Reproduction, Ministry of Education;

College of Animal Science and Veterinary Medicine, Huazhong Agricultural University, Wuhan, 430070, P. R. China

Received September 30, 2016; revised December 5, 2016; accepted June 23, 2017

Table S1. Monthly PRRSV positive rate of detection between 2012 and 2015

\begin{tabular}{|c|c|c|c|c|}
\hline Season & Month & No. of total & No. of positive & Positive rate \\
\hline \multirow{3}{*}{ Winter } & December & 1317 & 744 & $56.49 \%$ \\
\hline & January & 904 & 579 & $64.05 \%$ \\
\hline & February & 682 & 410 & $60.12 \%$ \\
\hline \multicolumn{2}{|c|}{ Three months total (winter) } & 2903 & 1733 & $59.70 \%$ \\
\hline \multirow{3}{*}{ Spring } & March & 1586 & 989 & $62.36 \%$ \\
\hline & April & 1249 & 671 & $53.72 \%$ \\
\hline & May & 1164 & 511 & $43.90 \%$ \\
\hline \multicolumn{2}{|c|}{ Three months total (spring) } & 3999 & 2171 & $54.29 \%$ \\
\hline \multirow{3}{*}{ Summer } & June & 4681 & 2581 & $55.14 \%$ \\
\hline & July & 1192 & 645 & $54.11 \%$ \\
\hline & August & 1111 & 575 & $51.76 \%$ \\
\hline \multicolumn{2}{|c|}{ Three months total (summer) } & 6984 & 3801 & $54.42 \%$ \\
\hline \multirow{3}{*}{ Autumn } & September & 1250 & 657 & $52.56 \%$ \\
\hline & October & 1095 & 601 & $54.89 \%$ \\
\hline & November & 1296 & 848 & $65.43 \%$ \\
\hline \multicolumn{2}{|c|}{ Three months total (autumn) } & 3641 & 2106 & $57.84 \%$ \\
\hline
\end{tabular}


Table S2. Geographical PRRSV positive rate of detection between 2012 and 2015

\begin{tabular}{llcccccccc}
\hline Regions & & Northeast & North China & East China & South China & Central China & Southwest & Northwest & Total \\
\hline \multirow{3}{*}{2012} & No. of total & 42 & 37 & 410 & 135 & 1645 & 172 & 51 & 2492 \\
& No. of positive & 29 & 31 & 231 & 95 & 686 & 42 & 24 & 1138 \\
& Positive rate & $69.05 \%$ & $83.78 \%$ & $56.34 \%$ & $70.37 \%$ & $41.70 \%$ & $24.42 \%$ & $47.06 \%$ & $45.67 \%$ \\
\hline \multirow{2}{*}{2013} & No. of total & 162 & 224 & 376 & 184 & 2211 & 54 & 213 & 3424 \\
& No. of positive & 108 & 109 & 260 & 143 & 1201 & 39 & 57 & 1917 \\
& Positive rate & $66.67 \%$ & $48.66 \%$ & $69.15 \%$ & $77.72 \%$ & $54.32 \%$ & $72.22 \%$ & $26.76 \%$ & $55.99 \%$ \\
\hline \multirow{2}{*}{2014} & No. of total & 24 & 249 & 866 & 324 & 2318 & 46 & 88 & 3915 \\
& No. of positive & 13 & 123 & 594 & 235 & 1198 & 30 & 35 & 2228 \\
& Positive rate & $54.17 \%$ & $49.40 \%$ & $68.59 \%$ & $72.53 \%$ & $51.68 \%$ & $65.22 \%$ & $39.77 \%$ & $56.91 \%$ \\
\hline \multirow{2}{*}{2015} & No. of total & 18 & 161 & 772 & 468 & 2015 & 97 & 205 & 3736 \\
& No. of positive & 11 & 95 & 458 & 241 & 1191 & 65 & 146 & 2207 \\
& Positive rate & $61.11 \%$ & $59.01 \%$ & $59.33 \%$ & $51.50 \%$ & $59.11 \%$ & $67.01 \%$ & $71.22 \%$ & $59.07 \%$ \\
\hline \multirow{2}{*}{ Three years } & No. of total & 246 & 671 & 2424 & 1111 & 8189 & 369 & 557 & 13567 \\
& No. of positive & 161 & 358 & 1543 & 714 & 4276 & 176 & 262 & 7490 \\
& Positive rate & $65.45 \%$ & $53.35 \%$ & $63.66 \%$ & $64.27 \%$ & $52.22 \%$ & $47.70 \%$ & $47.04 \%$ & $55.21 \%$ \\
\hline
\end{tabular}

